\title{
Transparency, swelling and scarring in the corneal stroma
}

KM Meek', DW Leonard², CJ Connon', S Dennis' and S Khan'

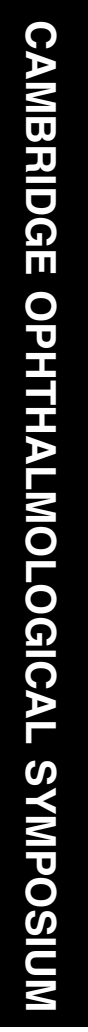

One of the most remarkable properties of the cornea is its ability to transmit almost all the incident light in the visible part of the spectrum. The reasons for corneal transparency have occupied scientists for many decades and despite considerable advances in our understanding, to date there is still no universally accepted explanation. Even more perplexing are the causes of increased light scattering in the cornea during wound healing or in some pathological situations.

In this paper, we briefly review some of the theories put forward to explain corneal transparency and use the most well tested of these to try to model the light scattering expected from oedematous corneas and from corneas following phototherapeutic keratectomy (PTK).

\section{Corneal transparency}

Any system where the attenuation of light is only caused by scattering (in other words there are no other losses such as might be due, for example, to absorption) can be described by

$$
F_{\mathrm{t}}=\exp \left(-\alpha_{\mathrm{s}} t\right)
$$

where $F_{\mathrm{t}}$ is the percentage of the incident light transmitted without scattering, $\alpha_{\mathrm{s}}$ is the scattering attenuation coefficient, and $t$ is the
${ }^{1}$ Cardiff Institute of Tissue Engineering and Repair and Department of Optometry and Vision Sciences Cardiff University

Cardiff, UK

${ }^{2}$ The Open University Oxford Research Unit Oxford, UK

Correspondence:

KM Meek

Cardiff Institute of Tissue Department of Optometry and Vision Sciences Cardiff University Redwood Building, Cardiff CF10 3NB, UK Tel: +442920876317 Fax: + 442920874859 E-mail: Meekkm@ cf.ac.uk

Received: 28 February 2003 Accepted in revised form: 28 February 2003

This work was funded by the Wellcome Trust and is currently funded by the Medical Research Council Engineering and Repair and
Lamellar interweave in the anterior stroma probably limits the extent to which the 
thickness in the direction of the light path. In the case of a corneal lamella consisting of parallel collagen fibrils, the scattering attenuation coefficient can be written as the product $\rho \sigma$, where $\rho$ is the number of fibrils per unit area in a cross-section (often called the bulk fibril number density or simply the number density) and $\sigma$ is the scattering cross-section. Over the years there have been many models put forward to explain transparency; the difference between these models essentially depends on the mathematical formulation of the scattering crosssection term. Here we describe the most important of these models. All must consider the structure of the cornea, that is, the size and shape of the stromal constituents and their refractive indices since each of these factors influences the amount of light scattered by the structure. In particular, the refractive index of the collagen fibrils, the refractive index of the extrafibrillar material, and the ratio of these two refractive indices, all play a major role in determining the extent of light scattered by the stroma.

The simplest model $^{1}$ proposes that all corneal components have a uniform refractive index (which is equivalent to a zero value for the scattering crosssection). This essentially means that light cannot distinguish between fibrils and the material between them, hence it can propagate directly through the tissue unscattered. This model is generally rejected, partly because it fails to explain two important properties of the cornea, birefringence and transparency loss when the structure is distorted. Also, recent X-ray diffraction data have unambiguously confirmed earlier evidence for a difference in the refractive indices of the collagen fibrils and of the extrafibrillar material. ${ }^{2}$

Most modern models are based on the lattice theory put forward by Maurice. ${ }^{3}$ By approximating the collagen fibril to perfect, infinitely long cylinders, an estimate of the scattering from an individual fibril can be calculated. The refractive index difference between the fibrils and interfibrillar matrix means that each fibril scatters a small amount of light. However, if the fibrils are packed in a lattice arrangement, correlation in their relative positions leads to destructive interference of light scattered away from the forward direction, all the light energy going into the constructive interference in the forward direction. However, both electron microscopy and X-ray diffraction do not show the presence of this regular packing of collagen fibrils., ${ }^{4,5}$

Table 1 shows these two models alongside the other main models, which are all based on Maurice's early work. Hart and Farrell ${ }^{4}$ showed that only short-range order in the positions of the collagen fibrils is necessary for the required destructive interference of scattered photons. Results from X-ray diffraction showed that the type of short-range order in the packing seen in electron
Table 1 Models to explain corneal transparency

\begin{tabular}{ll}
\hline Perfect crystal lattice & Maurice $(1957)^{3}$ \\
Uniform refractive index & Smith $(1969)^{1}$ \\
Short-range order & Hart and Farrell (1969) \\
Average area/fibril=correlation area & Benedek $(1971)^{8}$ \\
Perturbed lattice & Feuk $(1971)^{6}$ \\
Hard-core fibrils with PG coating & Twersky $(1975)^{7}$ \\
\hline
\end{tabular}

micrographs is indeed what is found in the tissue. ${ }^{5}$ Feuk ${ }^{6}$ developed a long-range order model based on small, random displacements of the fibrils from ideal lattice sites. Twersky, ${ }^{7}$ assuming that the fibrils were arranged as in a two-dimensional fluid, expressed the distribution explicitly in terms of the volume fraction occupied by the fibrils. Benedek ${ }^{8}$ considered the problem from the point of view of fluctuations in the fibril number density. These concepts were explored quantitatively by Vaezy and Clark, ${ }^{9}$ who examined fluctuations in the spatial arrangement of the collagen fibrils using Fourier methods. Recently, Ameen et $a l^{10}$ used photonic band structure methods to explain light transmission through corneal lattices. Space is too limited to go into these models in greater detail, so, for a fuller account, the reader is directed to reviews by Farrell and McCally ${ }^{11}$ and Freegard. ${ }^{12}$ A more generalised mathematical review of transparency in biological tissues is by Tuchin. ${ }^{13}$

By way of a summary, it has been pointed out by Farrell and McCally ${ }^{11}$ that all currently viable transparency theories agree with three points:

1. each fibril is an ineffective scatterer;

2. despite this, the large number of fibrils requires that destructive interference of scattered light must occur; and

3. the cornea is thin.

\section{Direct summation of fields method}

In 1986, Freund et al $^{14}$ published a method to compute light scattering from the cornea, following on from the theoretical principles previously advanced by Hart and Farrell. ${ }^{4}$ The technique can be used to predict transmission by an arbitrary short-range order distribution of different-sized fibrils. A full account of the approach, called the direct summation of fields (DSF) method, is found in the original papers. ${ }^{14,15}$ It is a statistical technique in which the scattering from each individual fibril is computed, then the effects of interference are included and summed for the whole tissue using a method called ensemble averaging. It is worth mentioning at this stage that, in this method, transmission is computed as a function of wavelength, ignoring the lamellar structure of the stroma and also the 
presence of stromal cells. With these assumptions in mind, however, the DSF method has been tested and found to give reliable results in a number of situations. $^{16-18}$

In order to use DSF to compute the expected light transmission, it is necessary to measure a number of structural and physical properties of the stroma. The refractive index of the hydrated collagen fibrils, the refractive index of the interfibrillar matrix, and the ratio of these have previously been obtained using X-ray diffraction measurements in a number of different species. ${ }^{2}$ The relative positions of the individual fibrils, and the diameter of each fibril, are obtained from electron micrographs. There is a problem in using measurements from electron microscopy in that it has been shown that a number of microscope preparation protocols result in considerable shrinkage of fibril diameters and, particularly, interfibril spacings. ${ }^{19}$ To overcome this problem, we have used X-ray diffraction (a technique where corneas can be examined without the need for any processing ${ }^{20}$ ) to measure the mean values of these parameters in the same tissue as used for microscopy, and then scaled the data from electron micrographs so as to compensate for this shrinkage. Table 2 gives the values for several of these parameters for human corneas. The corneas were obtained from the Eye Bank in culture medium, and were dehydrated to close to physiological hydration using polyethylene glycol. ${ }^{21}$ It should be noted that the value for the fibril number density is lower than previously reported, partly owing to the scaling procedure.

Fibril diameters and spacings were measured by image analysis of selected electron micrographs where collagen fibrils were sectioned in cross-section. The positions and diameters of collagen fibrils in a human cornea were obtained after image analysis of these micrographs and scaled using X-ray data from the same samples as described above. By taking the corneal thickness as $0.52 \mathrm{~mm}$ and combining these data with the refractive index data in Table 2, the DSF method was used to predict the transmission as a function of wavelength. As expected (Figure 1), transmission was predicted to exceed $90 \%$ throughout most of the visible spectrum.

In their original paper ${ }^{4}$ and later ${ }^{16,15,11}$ Farrell and coworkers made only limited reference to how changes in

Table 2 Data from normal human cornea (relevant parameters were used in the direct summation of fields method to predict light transmission (Figure 1))

\begin{tabular}{lc}
\hline Average fibril diameter & $30.8 \pm 0.8 \mathrm{~nm}$ \\
Refractive index of fibrils & $1.411 \pm 0.001$ \\
Refractive index of extrafibrillar matrix & $1.365 \pm 0.003$ \\
Refractive index ratio & $1.033 \pm 0.002$ \\
Number density & $292 \pm 70 \mu \mathrm{m}^{2}$ \\
\hline
\end{tabular}

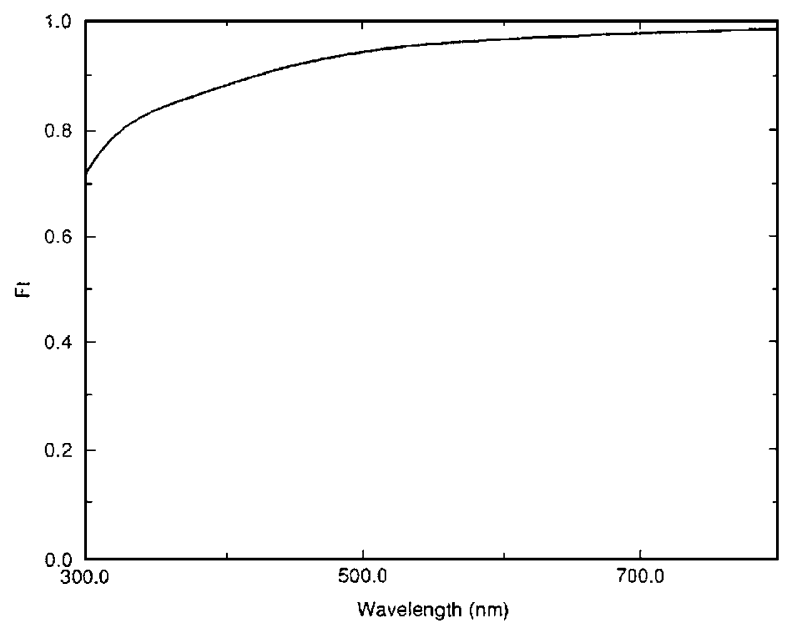

Figure 1 The summation of scattered fields method was used to predict transmission $\left(F_{\mathrm{t}}\right)$ as a function of wavelength in the human cornea. Data on corneal fibril positions and sizes were obtained from electron micrographs and were scaled to account for shrinkage during specimen preparation. Other data were taken from Table 2 .

the individual parameters might affect transparency in abnormal conditions. It is of interest to examine the theoretical effects of changing each of these parameters, keeping the rest constant, so as to gauge to which parameters light transmission is most sensitive. It should be remembered that here we are testing a single model for transparency, which has certain implicit assumptions about the tissue. Note also that this is a theoretical situation; in practice, many of the parameters are related, and a change in one is often accompanied by a change in one or more of the others. With these caveats, Figure 2a shows that increasing the fibril radius to $20 \mathrm{~nm}$ reduces transmission, particularly in the blue end of the spectrum, whereas reducing the radius has the opposite effect. So why not have small collagen fibrils in humans, as is found in fish? The answer is probably down to tissue mechanics - larger fibrils mean a stronger cornea.

The independent effect of the fibril number density on light scattering is difficult to assess, as altering the fibril number density by increasing the separation of the fibrils simultaneously changes the effects of interference. However, we find that keeping the relative positions of the fibrils constant but moving the fibrils apart (Figure 2b) leads to less scattering (greater transmission). An important point to realise, however, is that, contrary to what is often asserted, increased interfibril spacings (for example, when the cornea swells) are not per se responsible for the increased light scattering that accompanies oedema.

From Equation (1) it is clear that light scattering will increase with increased corneal thickness (assuming the increased thickness is because of extra tissue mass rather 
a

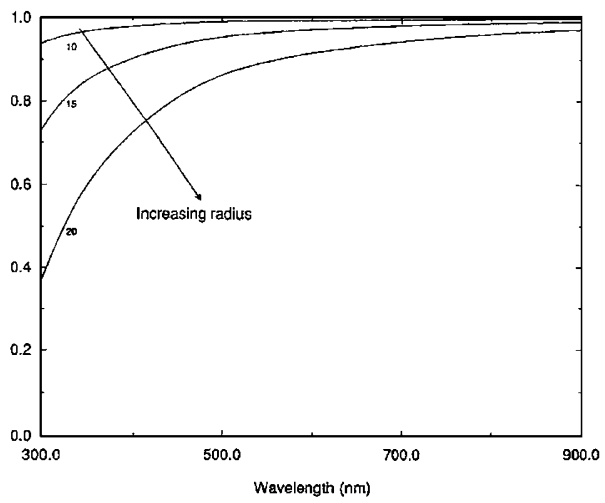

C

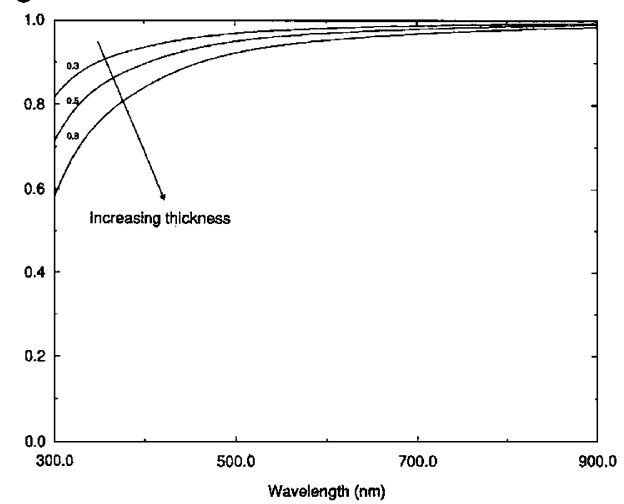

b

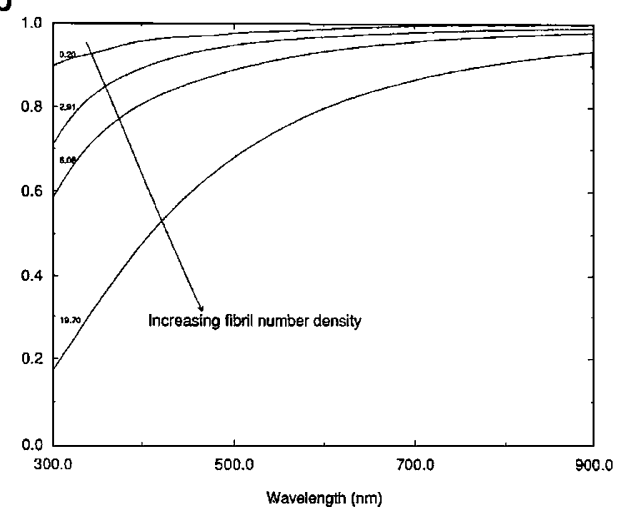

d

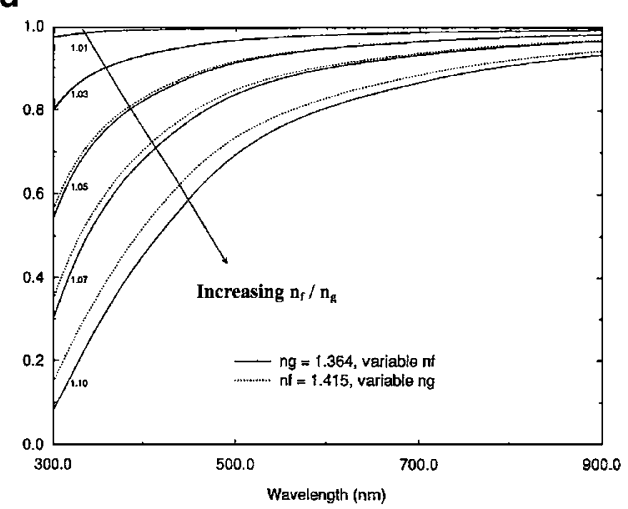

Figure 2 Theoretical effects of varying individual parameters as predicted by the summation of scattered fields model. The predicted transmission of light $\left(F_{\mathrm{t}}\right)$ is plotted against wavelength. The number by each curve represents the values of the parameters being varied, in units of $\mathrm{nm}(\mathrm{a}), \mu \mathrm{m}^{-2} \times 10^{-2}(\mathrm{~b})$, and $\mathrm{mm}(\mathrm{c})$.

than oedema). However, Figure $2 \mathrm{c}$ shows that this effect is relatively small, the corneal thickness could almost double without seriously increasing scattering. This presumably accounts for why, for example, a bovine cornea has similar transparency to a thinner human cornea.

Light transmission through the cornea is very sensitive to an increased mismatch in the refractive indices of the collagen and the extrafibrillar matrix. Theoretically, there are two ways of varying their ratio, either keep the refractive index of the fibrils constant and vary that of the matrix, or vice versa. Both have a similar effect (Figure $2 \mathrm{~d})$. If the ratio is one, there is total transmission throughout the spectrum. This is the uniform refractive index condition. As we increase the ratio, transmission reduces, once again, particularly at the blue end of the spectrum.

In conclusion, the DSF method can be used to demonstrate that light scattering in the cornea will increase if:

1. order in the spatial arrangement of the fibrils is destroyed;
2. fibril diameters increase;

3. fibril number density increases;

4. there is an increased refractive index imbalance between the hydrated fibrils and the extrafibrillar matrix;

5. corneal thickness increases.

So far, we have imagined the cornea as a structure made only of collagen fibrils and extrafibrillar matrix. Of course, there are a large number of keratocytes in the stroma, which gradually reduce in density from the anterior to the posterior stroma. ${ }^{22,23}$ Maurice $^{3}$ believed that there were insufficient of these to contribute significantly to scattering. Besides, they are relatively thin in the direction of the light path through the cornea. More recently, Jester $e t a l^{24}$ have suggested that these cells contain special proteins called corneal crystallins, which produce a uniform refractive index in the cells and may match the refractive index of the cytoplasm to that of the surrounding matrix. This, together with the dimensions of the cells, renders them weak scatterers (except for their nuclei, which are readily visible in the confocal microscope). However, if keratocytes change their shape 
or spill their contents, a different situation ensues, and they are capable of becoming very efficient scatterers. ${ }^{25,26}$

\section{Cornea oedema}

An understanding of structure and transparency changes when the cornea swells is dependent on our knowledge of where imbibed water is situated, both at the level of the tissue as a whole and within the lamellae themselves.

In many animals, the anterior stroma is less ordered, ${ }^{15}$ less hydrated, ${ }^{27-29}$ has a higher keratocyte density, ${ }^{22,23}$ has a lower keratan sulphate (KS) to chondroitin/ dermatan sulphate (DS) ratio, ${ }^{28}$ and is less easily swollen $^{29-31}$ than the posterior stroma. We have examined four frozen human corneas (two at physiological hydration and two swollen in culture medium). These were sectioned at $100 \mu \mathrm{m}$ intervals from the anterior to posterior using a Mikrom sliding microtome. All sections were weighed and then placed in $\mathrm{dH}_{2} \mathrm{O}$. At fixed intervals, each section was reweighed and then returned to the $\mathrm{dH}_{2} \mathrm{O}$ to continue swelling until a constant weight was reached. The hydration of each section was calculated for both the physiological and the swollen corneas. The results (Figure 3 ) confirm results from other species ${ }^{27-29}$ and show that hydration increases with tissue depth in both the physiological and the swollen human corneas. This may be related to the gradual increase in the KS/DS ratio with depth, since KS is known to show greater water absorption than DS. ${ }^{32,33}$ However, as Bron ${ }^{34}$ has pointed out it is possible that there is a differential loss of proteoglycans between

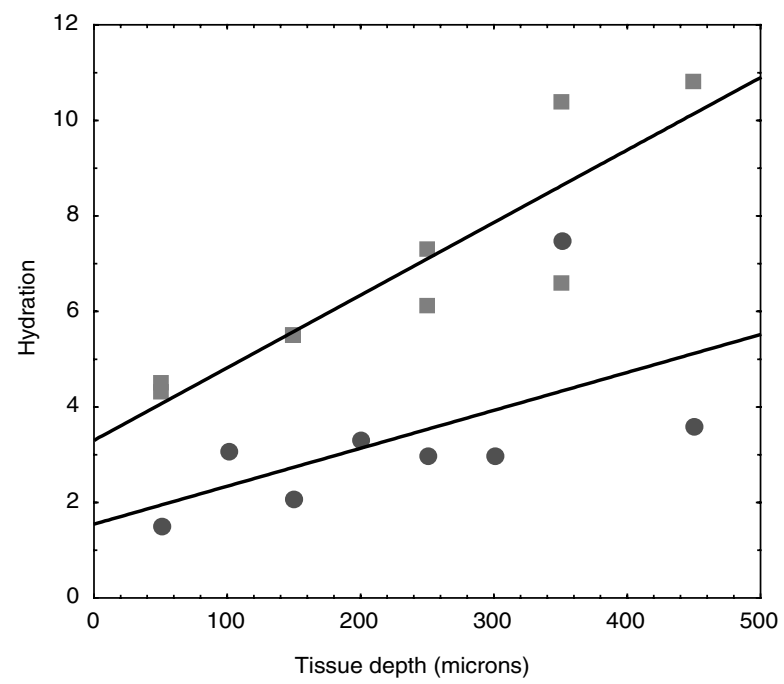

Figure 3 Hydration plotted as a function of stromal depth in the normal and swollen human cornea. (Circles) data from two corneas at about $H=3$; (squares) data from two corneas at about $H=7$. anterior and posterior stroma as the cornea swells and this, if it happens, would affect the swelling at different stromal depths.

The corneal sections were immersed in distilled water until they essentially stopped swelling. The final hydrations achieved were plotted as a function of tissue depth (Figure 4). We found that the maximum achievable hydration increases as a function of depth. This means that the posterior stroma is capable of swelling much more than the anterior. It is likely that anterior swelling is limited by lamellar interweaving and insertions into Bowman's layer, a phenomenon that may have considerable importance in maintaining the correct shape of the cornea. ${ }^{31,34}$

Information on the distribution of imbibed water within the lamellae has been obtained using X-ray diffraction methods. When the denuded cornea swells, there is a linear relation between the fibril separation squared and the hydration. ${ }^{35}$ Interfibrillar centre-to-centre spacings were determined for bovine corneas as a function of hydration using $\mathrm{X}$-ray diffraction, and the results are shown in Figure 5 (line a). By extrapolating this line to $H=0$, it is possible to plot a theoretical graph of the expected interfibril spacing on the assumption that all the water entering the stroma has gone towards separating the constituent fibrils. ${ }^{5,21}$ This theoretical plot is shown in Figure 5 (line b). The shading around the theoretical plot is the uncertainty owing to the uncertainty in determining the spacing at $H=0$ from the experimental data. The interesting point is that at aiven hydration, the interfibril spacing is lower than it should be considering the amount of water in the stroma. This must mean that some of the water is not between fibrils, and thus must be in fibril-free regions. Some of these regions are probably places occupied by cells that have died post mortem. If we assume that keratocytes occupy

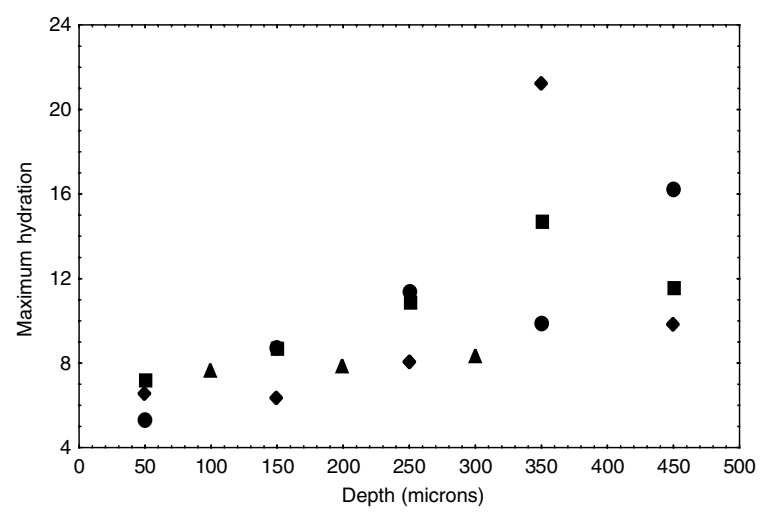

Figure 4 The final hydration achieved in four human corneas plotted as a function of stromal depth. (Circles) 72-year-old male subject; (squares) 82-year-old female subject; (diamonds) 91-year-old male subject; (triangles) 79-year-old male subject. 


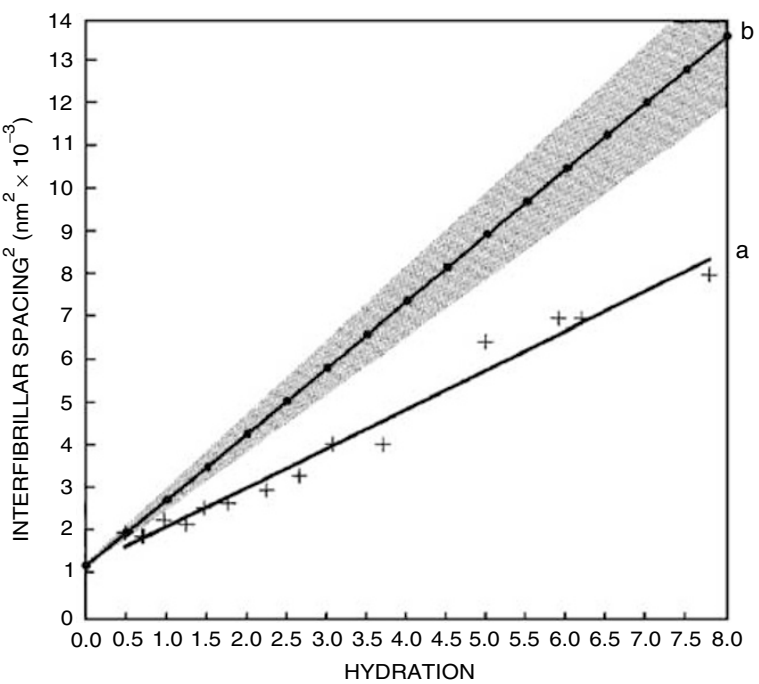

Figure 5 Graph (a) shows the variation of the centre-to-centre collagen fibril spacing (squared) plotted as a function of tissue hydration. The data were obtained using low-angle synchrotron X-ray diffraction. Graph (b) is the theoretical centre-to-centre fibril spacing on the assumption that all the fluid entering the stroma is distributed evenly between the collagen fibrils. The shaded area represents the uncertainty in line (b) owing to the uncertainty in determining the intersect of line (a) at $H=0$. Reproduced from Meek $e t a l^{21}$ with permission.

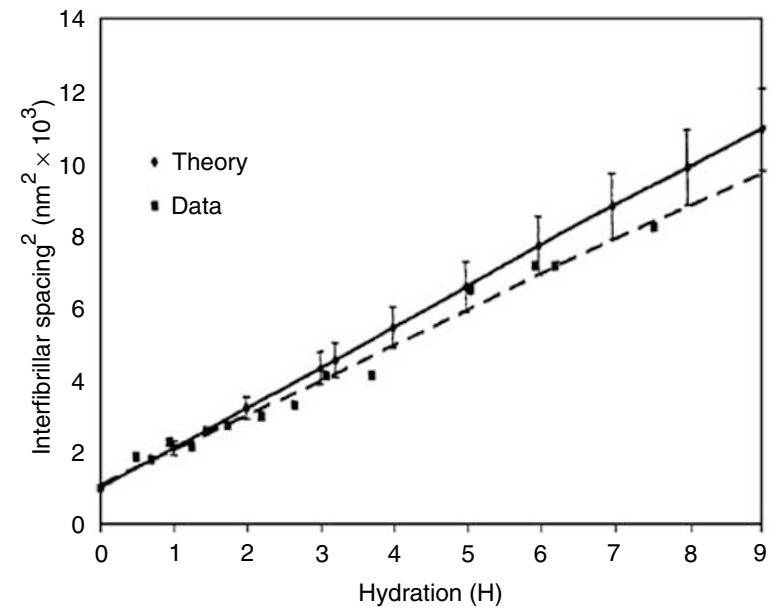

Figure 6 The lower line shows the data from line (a) in Figure 5. The upper line shows the theoretical centre-to-centre collagen fibril spacing on the assumption that $15 \%$ of the fluid entering the stroma goes into fibril-free regions ('lakes'). Reproduced from Huang and $\mathrm{Meek}^{36}$ with permission.

$15 \%$ of the stromal volume, we can take this into account in the theoretical calculation, and the match between theory and experiment becomes much better (Figure 6).

So it appears that fibril-free regions ('lakes') form in swollen corneas, and that when cells die, the spaces previously occupied by them might themselves become 'lakes' that could contribute to an increase in light scattering.

\section{Light scattering in oedematous corneas}

A full survey of the literature probing the causes of scattering when the cornea swells is beyond the scope of this article. From a theoretical standpoint, 'lakes' would add a term to the total scattering cross-section that would vary as $B / \lambda^{2} \cdot{ }^{37,38}$ By measuring transmission as corneas swelled, Farrell et al were able to compute the scattering cross-section and demonstrated that it has a $1 / \lambda^{2}$ dependence, as predicted by the presence of 'lakes' ${ }^{35}$ Lakes are not seen in the normal human cornea, but they are present in bullous keratopathy and Fuch's dystrophy corneas. ${ }^{39}$ Some fibril-free regions appear to be because of matrix disorder (Figure 7), while others might reflect the presence of dead cells. The question is, to what extent does the intralamellar disordering lead to light scattering?

In principle, the summation of scattered fields approach could again be used to compute the theoretical effects of disordering and/or lakes. The required structural information (fibril positions, diameters, number density) can be obtained from electron micrographs scaled according to the $\mathrm{X}$-ray diffraction measurements as described previously. However, as the cornea swells, the extra water will change the refractive indices of the interfibrillar matrix and also, possibly, of the collagen fibrils themselves. However, we know from previous studies ${ }^{21}$ that collagen fibrils do not swell appreciably above physiological hydration, so their refractive index is independent of tissue hydration and stays constant at 1.416. As water or electrolyte enters the interfibrillar matrix, it dilutes it and the refractive index falls. The amount by which it falls can be estimated by measuring the change in the refractive index of the stroma as a function of tissue thickness or hydration (Figure 8) and then applying Gladstone and Dales's law of refractive indices to the system. ${ }^{2}$ Since the imbibed fluid does not enter the fibrils themselves ${ }^{21}$ this fall in the refractive index as the stroma swells leads to an increase in the ratio of the refractive index of the fibrils to that of the interfibrillar matrix and to a corresponding increase in light scattering. We found that between physiological hydration and $H=3.8$, there was a $0.15 \%$ reduction in the refractive index of the matrix and a corresponding $0.1 \%$ increase in the ratio of the refractive indices of the fibrils and the matrix (S Khan, S Dennis, and K Meek, unpublished results). Between physiological hydration and $H=5.8$, these percentages were $0.59 \%$ and $0.58 \%$, respectively. 
a

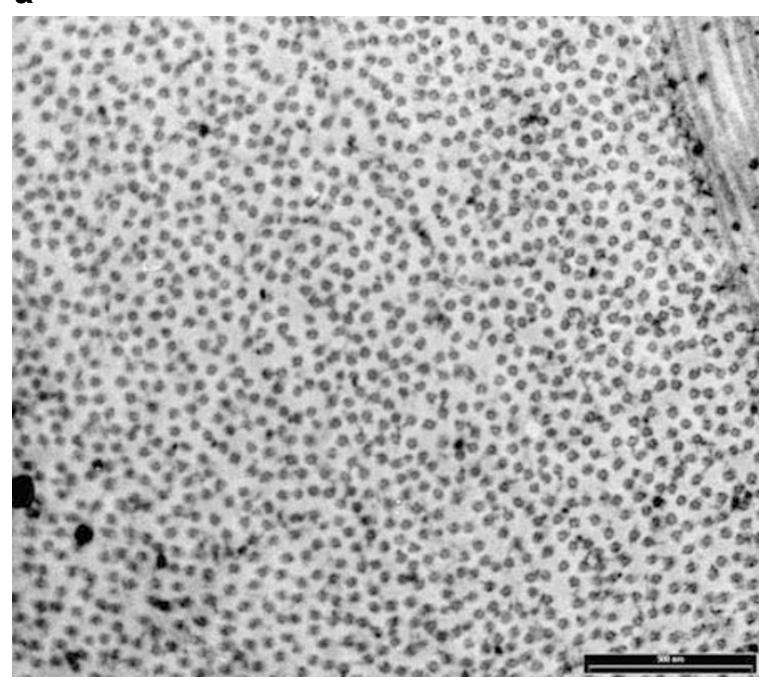

\section{b}

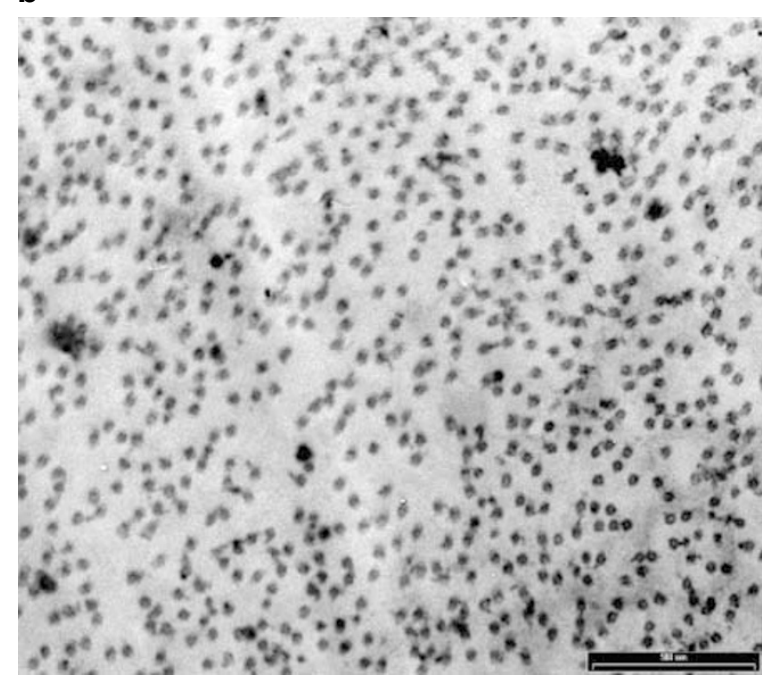

Figure 7 Electron micrographs from human corneas: (a) normal cornea $(H=3.8$; bar $=500 \mathrm{~nm})$. (b) Fuch's dystrophy cornea $(H=5.8$; bar $=500 \mathrm{~nm})$

Armed with this quantitative information, it is now possible to apply the summation of scattered fields method to Fuch's dystrophy corneas. The result is shown in Figure 9. It can be appreciated that the intralamellar disruption in the spatial arrangement of fibrils, the increased mismatch in the refractive indices, and the increased thickness of the stroma together lead to the overall reduction in light transmission.

\section{Phototherapeutic keratectomy}

It is well known that haze develops following laser ablation to the anterior stroma. The definition of haze is difficult because of the different methods used to

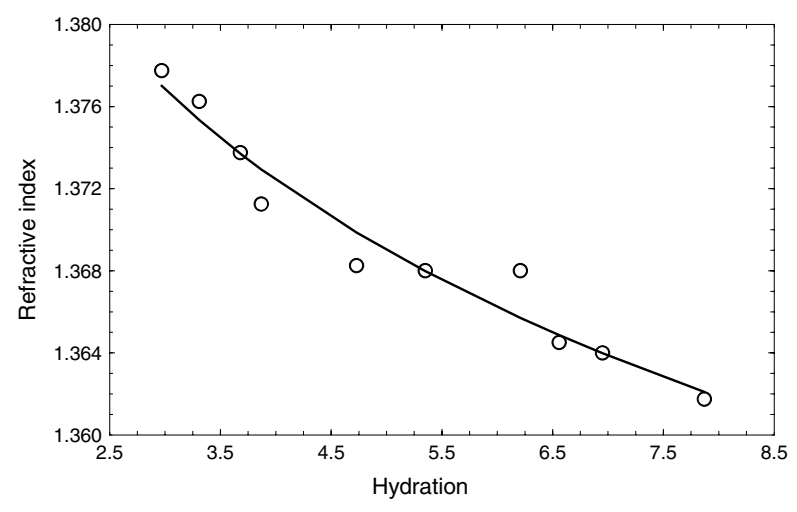

Figure 8 Refractive index of the human cornea plotted as a function of tissue hydration. The hydration of the stroma was altered by dialysing the denuded stroma against phosphate buffer $\mathrm{pH} 7.1^{36}$ to which different amounts of the osmotic agent polyethylene glycol were added. ${ }^{21}$ The continuous line is a leastsquares best fit to the data points.

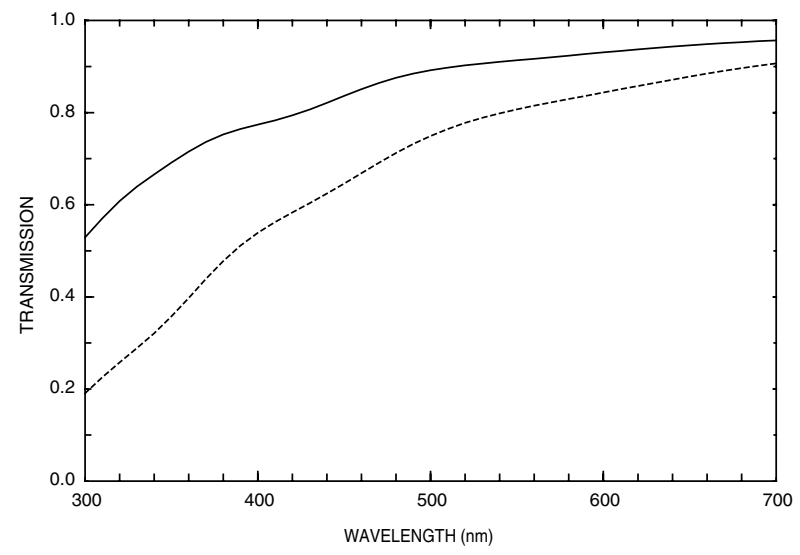

Figure 9 The Summation of Scattered Fields method was used to predict transmission as a function of wavelength in the swollen human corneas. (Continuous line) normal; (broken line) Fuch's dystrophy.

measure it, and even less certain is the origin of the haze. ${ }^{40}$ Various authors have ascribed the observed haze to irregularities in the epithelium, ${ }^{41,42}$ to subepithelial deposits, ${ }^{43}$ to the presence of vacuoles, ${ }^{44}$ to the deposition of poorly organised collagen, ${ }^{45,46}$ or to the presence of activated keratocytes. ${ }^{25,26}$ However, none of these suggestions have been experimentally or theoretically shown to account for increased light scattering. We have used PTK in rabbits to predict the percentage

transmission of visible light through the newly deposited collagen using the DSF method and hence to see if this collagen could account for the observed haze. All experimental procedures were carried out in accordance with the ARVO Resolution on the Use of animals in Ophthalmic and Vision Research. 
PTK took place at St Thomas' Hospital London using an Omnimed excimer laser (Summit Technology, Boston, MA, USA) with a wavelength of $193 \mathrm{~nm}$. The pulse energy resulted in a radiant exposure of $180 \mathrm{~mJ} / \mathrm{cm}^{2}$ at a pulse frequency of $10 \mathrm{~Hz}$. The beam shape was circular with a fixed diameter of $6.0 \mathrm{~mm}$. Wounds were allowed to heal for up to 19 months. We used an objective measurement for corneal haze developed by Lohmann et $a l^{47}$ in which haze was determined using a slit-lampmounted charged-coupled device (CCD) system. The results of the haze measurements are shown in Figure 10 and confirm that both a transitory haze (which peaks after a month) and a more persistent or late developing haze (which remains for many months) occur. The question we address here is to what extent the persistent haze can be ascribed to the nature of the newly deposited collagen.

After 8 months of healing, the rabbit corneas had laid down a layer of newly deposited collagen that had almost compensated for the amount removed (approximately $100 \mu \mathrm{m}$ ). Apart from the most superficial layer, most of this collagen had formed a lamellar structure, although the order in the fibril packing was visibly poor. Micrographs were taken at different depths and typical ones were used in the DSF method to predict light transmission. In this case, however, we had no information about refractive indices in the new matrix, so we made the assumption that these were normal.

Figure 11 shows that despite the fact that the fibril diameters and organisation had not returned to normal, only a very small drop in light transmission is predicted. This is probably due, in large part, to the fact that the newly deposited layer extended to only about $100 \mu \mathrm{m}$. The thinness of this collagenous layer, therefore, counteracts the increased scattering caused by the poor organisation of the new collagen. It appears, therefore,

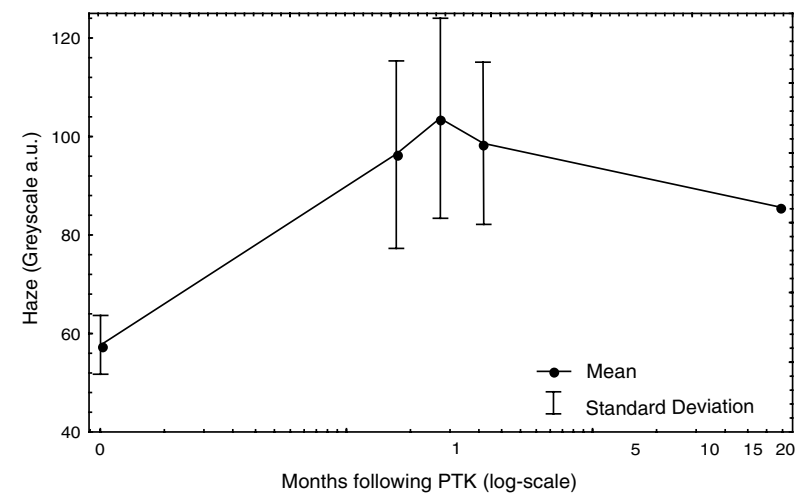

Figure 10 Development of haze as a function of time during wound healing in rabbit corneas. Reproduced from Connon et $a l^{48}$ with permission.

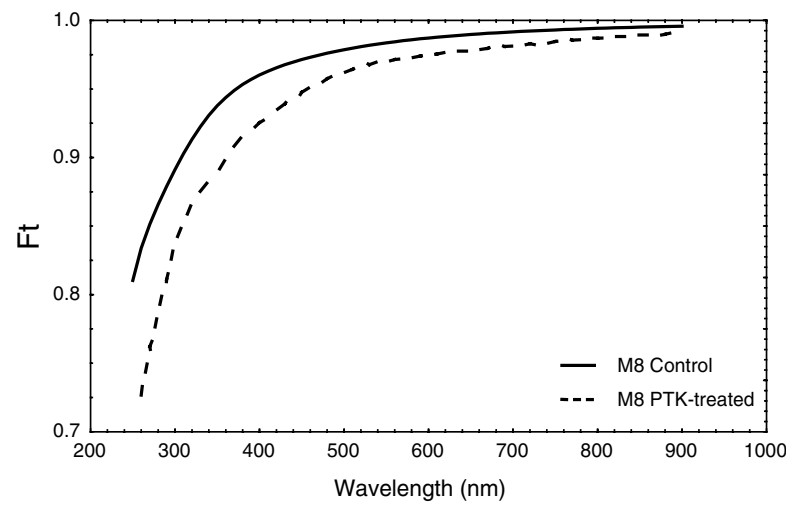

Figure 11 The Summation of Scattered Fields method was used to predict transmission as a function of wavelength in a rabbit cornea 8 months after PTK and in its control cornea. Reproduced from Connon et al ${ }^{48}$ with permission.

that newly deposited collagen is not the cause of persistent haze following PTK. Electron microscopy of our rabbit corneas showed a qualitative correlation between haze, the number of activated keratocytes, and the smoothness of the subepithelial basement lamina. We therefore believe that either or both of these contribute more to post-PTK persistent haze than does the newly deposited collagen.

\section{Conclusions}

Despite the considerable effort that has been put into understanding corneal transparency, there is still no universally accepted explanation and no model that has been thoroughly tested. Some progress has been made, particularly with respect to our understanding of what factors govern corneal fibril size ${ }^{49,50}$ and organisation, including the roles of the ambient ions ${ }^{51}$ and of proteoglycans. The recent availability of gene-targeted mice with null mutations for selected proteoglycans ${ }^{52-54}$ now makes it possible to correlate the structural effect of selected deletions with tissue transparency. For example, it is interesting that lumican-null mice have cloudy corneas, ${ }^{52}$ decorin-null mice have clear corneas, ${ }^{53}$ and keratocan-null mice have mostly clear corneas. ${ }^{54}$ These and similar tissues open the possibility to greatly increase our understanding of the causes of light scattering from abnormal corneas in the near future.

\section{Acknowledgements}

We thank Professor John Marshall and Anne Patmore for carrying out the PTK and making the haze measurements shown in Figure 10. We also thank Mr Nick Hawksworth for supplying postoperative 
pathological corneas, Dr Val Smith for supplying normal human corneas from the Bristol Eye Bank, and Dr S Akhtar for assistance with electron microscopy. We are grateful to the staff at the SRS Daresbury laboratory for their ongoing help with the data collection.

\section{References}

1 Smith JW. The transparency of the corneal stroma. Vis Res 1969; 9: 393-396.

2 Leonard DW, Meek KM. Refractive indices of the collagen fibrils and extrafibrillar material in the corneal stroma. Biophys J 1997; 72: 1382-1387.

3 Maurice DM. The structure and transparency of the corneal stroma. J Physiol 1957; 136: 263-286.

4 Hart RW, Farrell RA. Light scattering in the cornea. J Opt Soc Am 1969; 59: 766-774.

5 Sayers Z, Koch MHJ, Whitburn SB, Meek KM, Elliott GF, Harmsen A. Synchrotron X-ray diffraction study of the corneal stroma. J Mol Biol 1982; 160: 593-607.

6 Feuk T. On the transparency of the stroma in the mammalian cornea. IEEE Trans Biomed Eng 1970; BME17 1866-1890.

7 Twersky V. Transparency of pair-correlated, random distributions of small scatterers, with applications to the cornea. J Opt Soc Am 1975; 65: 524-530.

8 Benedek GB. Theory and transparency of the eye. Appl Opt 1971; 10: 459-473.

9 Vaezy S, Clark JI. Quantitative analysis of the microstructure of the human cornea and sclera using 2-D Fourier methods. J Microsc 1994; 175: 93-99.

10 Ameen DB, Bishop MF, McMullen T. A lattice model for computing the transmissivity of the cornea and sclera. Biophys J 1998; 75: 2520-2531.

11 Farrell RA, McCally RL. Corneal transparency. In: Albert DM, Jakobiec FA (eds). Principles and Practice of Ophthalmology. WB Saunders: Philadelphia, PA, 2000, pp 629-643.

12 Freegard TJ. The physical basis of transparency of the normal cornea. Eye 1997; 11: 465-471.

13 Tuchin VV. Light scattering study of tissues. Phys Usp 1997; 40: 495-515.

14 Freund DE, McCally RL, Farrell RA. Direct summation of fields for light scattering by fibrils with applications to normal corneas. Appl Opt 1986; 25 : 2739-2746.

15 Freund DE, McCally RL, Farrell RA, Cristol SM, L'Hernault NL, Edelhauser HF. Ultrastructure in anterior and posterior stroma of perfused human and rabbit corneas: relation to transparency. Invest Ophthalmol Vis Sci 1995; 36: 1508-1523.

16 Freund DE, McCally RL, Farrell RA. Light scattering tests of structure in normal and swollen rabbit corneas. Johns Hopkins APL Tech Dig 1991; 12: 137-143.

17 Leonard DW. The ultrastructure of the corneal stroma and its implications for transparency. PhD thesis, The Open University, Milton Keynes, UK, 1996.

18 Rawe IM, Leonard DW, Meek KM, Zabel RW. X-ray diffraction and transmission electron microscopy of Morquio Syndrome Type A cornea: a structural analysis. Cornea 1997; 16: 369-376.
19 Fullwood NJ, Meek KM. A synchrotron X-ray study of the changes occurring in the corneal stroma during processing for electron microscopy. J Microsc 1993; 169: 53-60.

20 Meek KM, Quantock AJ. The use of X-ray scattering techniques to determine corneal ultrastructure. Prog Ret Eye Res 2001; 20: 95-137.

21 Meek KM, Fullwood NJ, Cooke PH, Elliott GF, Maurice DM, Quantock AJ et al. Synchrotron X-ray diffraction studies of the cornea with implications for stromal hydration. Biophys J 1991; 60: 467-474.

22 Patel S, McLaren J, Hodge D, Bourne W. Normal human keratocyte density and corneal thickness measurement by using confocal microscopy in vivo. Invest Ophthalmol Vis Sci 2001; 42: 333-339.

23 Moller-Pedersen T, Ehlers N. A three-dimensional study of the human corneal keratocyte density. Curr Eye Res 1995; 14: 459-464.

24 Jester JV, Moller-Pedersen T, Huang J, Sax CM, Kays WT, Cavangh HD et al. The cellular basis of corneal transparency: evidence for 'corneal crystallins'. J Cell Sci 1999; 112: 613-622.

25 Moller-Pedersen T, Cavanagh HD, Petroll WM, Jester JV. Stromal wound healing explains refractive instability and haze development after photorefractive keratectomy: a 1-year confocal microscopic study. Ophthalmology 2000; 107: 1235-1245.

26 Moller-Pedersen T. The cellular basis of corneal transparency and haze development (Abstract). Ophthalmic Res 2002; 34: 4

27 Turss R, Friend J, Reim M, Dohlman CH. Glucose concentration and hydration of the corneal stroma. Ophthalmic Res 1971; 2: 253-260.

28 Castoro JA, Bettelheim AA, Bettelheim FA. Water gradients across bovine cornea. Invest Ophthalmol Vis Sci 1988; 29: 963-968.

29 Lee D, Wilson G. Non-uniform swelling properties of the corneal stroma. Curr Eye Res 1981; 1: 457-461.

30 Kikkawa Y, Hirayama K. Uneven swelling of the corneal stroma. Invest Ophthalmol 1970; 9: 735-741.

31 Müller L, Pels E, Vrensen GFJM. The specific architecture of the anterior stroma accounts for maintenance of corneal curvature. Br J Ophthalmol 2001; 85: 437-443.

32 Plessy B, Bettelheim FA. Water vapor sorption of keratan sulfate. Mol Cell Biochem 1975; 6: 85-91.

33 Bettelheim FA, Plessy B: The hydration of proteoglycans of bovine cornea. Biochim Biophys Acta 1975; 381: 203-214.

34 Bron AJ. The architecture of the corneal stroma. $\mathrm{Br} \mathrm{J}$ Ophthalmol 2001; 85: 379-383.

35 Goodfellow JM, Elliott GF, Woolgar AE. X-ray diffraction studies of the corneal stroma. J Mol Biol 1978; 119: 237-252.

36 Huang Y, Meek KM. Swelling studies on the cornea and sclera: the effects of $\mathrm{pH}$ and ionic strength. Biophys J 1999; 77: 1655-1665.

37 Farrell RA, McCally RL, Tatham PER. Wavelength dependencies of light scattering in normal and cold swollen rabbit corneas and their structural implications. J Physiol (London) 1973; 233: 589-612.

38 Farrell RA, McCally RL. On corneal transparency and its loss with swelling. J Opt Soc Am 1976; 66: 342

39 Quantock AJ, Meek KM, Brittain P, Ridgway AE, Thonar EJ. Alteration of the stromal architecture and depletion of keratan sulphate proteoglycans in oedematous human corneas: histological, immunochemical and X-ray diffraction evidence. Tissue Cell 1991; 23: 593-606. 
40 Corbett MC, Marshall J. Corneal haze after photorefractive keratectomy, a review of etiological mechanisms and treatment options. Lasers Light 1996; 7: 173-196.

41 Tuft SJ, Marshall J, Rothery S. Stromal remodelling following photorefractive keratectomy. Lasers Ophthalmol 1987; 1: 177-183.

42 Marshall J, Trokel S, Rothery S, Krueger RR. Long term healing of the central cornea after photorefractive keratectomy using an excimer laser. Ophthalmology 1988; 95: 1411-1421.

43 Hanna KD, Pouliquen Y, Waring GO, Savoldelli M, Cotter J, Morton K et al. Corneal stromal wound healing after $193 \mathrm{~nm}$ excimer laser ablation. Arch Ophthalmol 1989; 107: 895-901.

44 Rawe IM, Zabel RW, Tuft SJ, Chen V, Meek KM. A morphological study of rabbit corneas after laser keratectomy. Eye 1992; 6: 637-642.

45 Fantes FE, Hanna KD, Waring III GO, Pouliquen Y, Thompson KP, Savoldelli M. Wound healing after excimer laser keratomileusis in monkeys. Arch Ophthalmol 1990; 108: 665-675.

46 Wu WCS, Stark WJ, Green WR. Corneal wound healing after 193 excimer laser keratectomy. Arch Ophthalmol 1991; 109: $1426-1432$.

47 Lohmann CP, Fitzke F, O'Brart D, Muir MK, Timberlake G, Marshall J. Corneal light scattering and visual performance in myopic individuals with spectacles, contact lenses, or excimer laser photorefractive keratectomy. Am J Ophthalmol 1993; 155: 444-453.
48 Connon CJ, Marshall J, Patmore AL, Brahma A, Meek KM. Persistent haze and disorganisation of anterior stromal collagen appear unrelated following phototherapeutic keratectomy (PTK). J Refract Surg 2003; 19: 1-11.

49 Adachi E, Hayashi T. In vitro formation of hybrid fibrils of type $\mathrm{V}$ collagen and type I collagen. Limited growth of type I collagen into thick fibrils by type $\mathrm{V}$ collagen. Connect Tissue Res 1986; 14: 257-266.

50 Birk DE. Type V collagen: heterotypic type I/V collagen interactions in the regulation of fibril assembly. Micron 2001; 32: 223-237.

51 Kostyuk O, Nalovina O, Mubard T, Reginio JW, Meek KM, Quantock AJ et al. Transparency of the bovine corneal stroma at physiological hydration and its dependence on concentration of the ambient anion. J Physiol 2002; 243(2): 633-642.

52 Chakravarti S, Petroll WM, Hassell JR, Jester JV, Lass JH, Paul J et al. Corneal opacity in lumican-null mice: defects in collagen fibril structure and packing in the posterior stroma. Invest Ophthalmol Vis Sci 2000; 41: 3365-3373.

53 Danielson KG, Baribault H, Holmes DF, Graham H, Kadler KE, Iozzo RV. Targeted disruption of decorin leads to abnormal collagen fibril morphology and skin fragility. J Cell Biol 1997; 136: 729-743.

54 Saika S, Shiraishi A, Liu CY, Funderburgh JL, Kao CW, Converse RL et al. Role of lumican in the corneal epithelium during wound healing. J Biol Chem 2000; 275: 2607-2612. 